\title{
Copolymer at a selective interface and two dimensional wetting: a grand canonical approach
}

\author{
C. Monthus *, T. Garel and H. Orland \\ Service de Physique Théorique \\ CE-Saclay, 91191 Gif-sur-Yvette Cedex, France
}

(October 26, 2018)

\begin{abstract}
We consider two different problems involving the localization of a single polymer chain: (i) a periodic $A B$ copolymer at a selective fluid-fluid interface, with the upper (resp. lower) fluid attracting $A$ (resp. $B$ ) monomers (ii) a homopolymer chain attracted to a hard wall (wetting). Self avoidance is neglected in both models, which enables us to study their localization transition in a grand canonical approach. We recover the results obtained in previous studies via transfer matrix methods. Moreover, we calculate in this way the loop length distribution functions in the localized phase. Some finite size effects are also determined and tested numerically.
\end{abstract}

Submitted for publication to: "Eur. Phys. J. B"

Saclay, SPhT/00-037

PACS: 05.70 Np; 61.41.+e; 64.70.-p; 68.45 Gd

*On leave from LPTMS, Bat.100, 91406 Orsay, France 


\section{INTRODUCTION}

The behaviour of polymers at interfaces is currently a subject of great activity 1 目. In this paper, we focus our interest on two problems, namely (i) the adsorption of a periodic copolymer chain on a selective fluid-fluid interface (ii) the adsorption of a homopolymer chain on a hard wall (wetting). These problems have been previously solved for one dimensional geometries, provided certain assumptions are fulfilled (self avoidance is neglected, wetting is considered in the SOS approximation,...). A quick guide to the recent litterature can be found in references 3 - 3 and references therein. Here, we will tackle these models, with the same assumptions as above, through the use of a grand canonical method. This method was initiated in the context of the DNA helix-coil transition 12 and was recently used in the context of RNA folding 13 . We will recover previously known results about models (i) and (ii). Moreover, this approach enables us to obtain the loop length distribution function in the localized regime. We also find in this way some analytic expressions of finite size corrections at the critical temperature, which are in agreement with numerical transfer matrix calculations. The lay out of the paper is as follows. Section \ deals with the periodic $A B$ copolymer chain at a selective interface. In section [II], we consider the wetting of a homogeneous wall by a homopolymer chain.

\section{PERIODIC COPOLYMER CHAIN AT A SELECTIVE INTERFACE}

\section{A. Introduction}

We consider a periodic $A B$ copolymer chain of $2 N$ monomers at a selective fluid-fluid interface, located at $z_{0}=\frac{1}{2}$. The upper (resp. lower) fluid is a good solvent for $A$-type (resp. $B$-type) monomers, and will herefrom be called fluid $\alpha$ (resp. fluid $\beta$ ). This situation is modelled through the Hamiltonian

$$
\mathcal{H}_{2 N}=-\sum_{i=1}^{2 N} q_{i} \operatorname{sgn}\left[z(i)-\frac{1}{2}\right]
$$

where $\operatorname{sgn}(u)$ denotes the sign function, and the "charges" $q_{i}$ of the periodic chain are chosen

as $q_{2 n+1}=q_{A}>0$ and $q_{2 n}=-q_{B}<0$, together with $q_{A} \geq q_{B}$. In equation (11), $z(i)$ denotes the position of monomer $i$ of the chain, which we represent as an (unidimensional) random walk (RW) with the properties $z(i)=. .,-2,-1,0,+1,+2, .$. , and $z(i+1)-z(i)= \pm 1$. We emphasize once more that self avoidance is neglected in this model.

The partition function then reads

$$
Z_{2 N}=\sum_{(R W)} e^{-\beta \mathcal{H}_{2 N}}
$$

where $\beta=\frac{1}{T}$ and the sum runs over all random walks (RW) starting with an $A$ monomer at $z(i=1)=1$. The thermodynamical properties of this model can be obtained through the use of transfer matrix method $\$ 5$, as given in Appendix A. Here, we follow references 12 , and consider a particular chain configuration as a collection of non interacting loops. This 
class of problems is solved in a grand canonical approach, by defining a grand canonical partition function

$$
Z(K)=\sum_{N=1}^{+\infty} K^{N} Z_{2 N}
$$

and noting that the radius of convergence $K_{*}(\beta)$ of the series $Z(K)$ gives the free energy per monomer in the thermodynamic limit

$$
f=\frac{\log \left(K_{*}(\beta)\right)}{2 \beta}
$$

Moreover, one can easily calculate in this way quantities such as the loop length distribution function, which are not accessible through the transfer matrix approach.

\section{B. Calculation of the grand canonical partition function}

The partition function $Z_{2 N}$ for a chain of $(2 N)$ monomers can then be decomposed according to the position $z(2 N)$ of the end point of the chain. For simplicity, we set $z(2 N)=$ $2 n$, and write

$$
Z_{2 N}=\sum_{n=-\infty}^{+\infty} Z_{2 N}(2 n)
$$

Given the expression of the Hamiltonian (1), the chain configurations can be further de-

composed into loops. A preliminary remark is as follows: if $\mathcal{N}(l, z)$ denotes the number of random walks of $l$ steps going from $z=0$ to $z \geq 0$ in the presence of an absorbing barrier at $z=0^{-}$, the image method 4 yields

$$
\begin{aligned}
& \mathcal{N}(2 l, 2 p)=C_{2 l}^{l+p}-C_{2 l}^{l+p+1} \\
& \mathcal{N}(2 l-1,2 p-1)=C_{2 l-1}^{l+p-1}-C_{2 l-1}^{l+p}
\end{aligned}
$$

depending on the parity of $(l, z)$.

Let us temporarily suppose that the chain end is in fluid $\alpha(2 n \geq 2)$. The first loop, located by hypothesis in fluid $\alpha$ contains $\left(2 l_{1}-1\right)$ monomers, namely $l_{1} \mathrm{~A}$ monomers and $\left(l_{1}-1\right) \mathrm{B}$ monomers. The second loop, located in fluid $\beta$, contains $\left(2 l_{2}-1\right)$ monomers, namely $\left(l_{2}-1\right)$ A monomers and $l_{2} \mathrm{~B}$ monomers.... The last loop is denoted by $l_{2 p}$. Finally, one must also specify the number $2 l$ of monomers between the interface and the chain end. We may then write

$$
\begin{aligned}
Z_{2 N}(2 n) & =\sum_{p=0}^{+\infty} \sum_{l_{1}=1}^{\infty} \sum_{l_{2}=1}^{\infty} \ldots \sum_{l_{2 p}=1}^{\infty} \sum_{l=1}^{\infty} \delta\left(2 N-\left(\sum_{i=1}^{2 p}\left(2 l_{i}-1\right)+2 l\right)\right) e^{\beta q_{B}+\beta\left(q_{A}-q_{B}\right) l_{1}} \mathcal{N}\left(2 l_{1}-2,0\right) \\
& e^{\beta q_{A}-\beta\left(q_{A}-q_{B}\right) l_{2}} \mathcal{N}\left(2 l_{2}-2,0\right) \ldots . . e^{\beta q_{A}-\beta\left(q_{A}-q_{B}\right) l_{2 p}} \mathcal{N}\left(2 l_{2 p}-2,0\right) \\
& e^{\beta\left(q_{A}-q_{B}\right) l} \mathcal{N}(2 l-1,2 n-1)
\end{aligned}
$$

Plugging equation (8) into equations (3) and (5), we get 


$$
Z(K)=\sum_{n=-\infty}^{+\infty} Z(K, 2 n)
$$

where

$$
\begin{aligned}
Z(K, 2 n) & =\sum_{N=1}^{+\infty} K^{N} Z_{2 N}(2 n) \\
& =\frac{1}{1-e^{\beta\left(q_{A}+q_{B}\right)} B\left(K^{1 / 2} e^{\beta q_{0}}\right) B\left(K^{1 / 2} e^{-\beta q_{0}}\right)} \sum_{l=1}^{\infty}\left(K e^{2 \beta q_{0}}\right)^{l} \mathcal{N}(2 l-1,2 n-1)
\end{aligned}
$$

with

$$
q_{0}=\frac{q_{A}-q_{B}}{2}
$$

and

$$
B(y)=\sum_{l=1}^{+\infty} y^{2 l-1} \mathcal{N}(2 l-2,0)=\frac{1}{2 y}\left(1-\sqrt{1-4 y^{2}}\right)
$$

We finally get, for $(2 n \geq 2)$

$Z(K, 2 n)=\sum_{N=1}^{+\infty} K^{N} Z_{2 N}(2 n)=\frac{1}{1-e^{\beta\left(q_{A}+q_{B}\right)} B\left(K^{1 / 2} e^{\beta q_{0}}\right) B\left(K^{1 / 2} e^{-\beta q_{0}}\right)}\left(\frac{B\left(K^{1 / 2} e^{\beta q_{0}}\right)}{K^{1 / 2} e^{\beta q_{0}}}-1\right)^{n}$

Similarly, if the chain end is in fluid $\beta(2 n \leq 0)$, we find

$Z(K, 2 n)=\sum_{N=1}^{+\infty} K^{N} Z_{2 N}(2 n)=\frac{e^{\beta\left(q_{A}+q_{B}\right)} B\left(K^{1 / 2} e^{\beta q_{0}}\right) B\left(K^{1 / 2} e^{-\beta q_{0}}\right)}{1-e^{\beta\left(q_{A}+q_{B}\right)} B\left(K^{1 / 2} e^{\beta q_{0}}\right) B\left(K^{1 / 2} e^{-\beta q_{0}}\right)}\left(\frac{B\left(K^{1 / 2} e^{-\beta q_{0}}\right)}{K^{1 / 2} e^{-\beta q_{0}}}-1\right)^{|n|}$

\section{Thermodynamics}

We finally get from (13) and (14)

$$
Z(K)=\sum_{n=-\infty}^{+\infty} Z(K, 2 n)=\frac{r_{+}\left(K^{1 / 2} e^{\beta q_{0}}\right)+e^{\beta\left(q_{A}+q_{B}\right)} B\left(K^{1 / 2} e^{\beta q_{0}}\right) r_{-}\left(K^{1 / 2} e^{-\beta q_{0}}\right)}{1-e^{\beta\left(q_{A}+q_{B}\right)} B\left(K^{1 / 2} e^{\beta q_{0}}\right) B\left(K^{1 / 2} e^{-\beta q_{0}}\right)}
$$

where

$$
\begin{aligned}
& r_{+}(y)=\frac{\frac{B(y)}{y}-1}{2-\frac{B(y)}{y}}=\frac{1}{2}\left(\frac{1}{\sqrt{1-4 y^{2}}}-1\right) \\
& r_{-}(y)=\frac{B(y)}{2-\frac{B(y)}{y}}=\frac{y}{\sqrt{1-4 y^{2}}}
\end{aligned}
$$

The radius of convergence $K_{*}(\beta)$ of the grand canonical partition function $Z(K)$ is either given by the singularity of the numerator of equation (15), or by the singularity of the denominator. The former corresponds to the value 


$$
K_{\text {deloc }}(\beta)=\frac{1}{4} e^{-2 \beta q_{0}}
$$

The latter comes from the resummation of the loops, and is given by

$$
K_{*}(\beta)=\frac{\sinh \left(\beta q_{A}\right) \sinh \left(\beta q_{B}\right)}{\left.\sinh ^{2} \beta\left(q_{A}+q_{B}\right)\right)}
$$

If the solution $K_{*}(\beta)$ is smaller than $K_{\text {deloc }}$ then the chain is localized. This actually happens for $\beta>\beta_{c}$ (i.e. $T<T_{c}$ ), where $\beta_{c}$ is the solution of

$$
e^{2 \beta_{c} q_{B}}+e^{-2 \beta_{c} q_{A}}-2=0
$$

In particular, in the limit $q_{0}=\left(q_{A}-q_{B}\right) / 2 \rightarrow 0$, the critical temperature $T_{c}$ diverges as

$$
T_{c} \underset{q_{0} \rightarrow 0}{\simeq} \frac{q_{A}^{2}}{q_{0}}
$$

Note that the scaling of $T_{c}$ with $q_{0}$ differs from equation (10) of reference ${ }^{7}$, since the model used in this reference allows the monomers to sit at the interface, in contrast to equation (10). The physics is nevertheless the same: a symmetric copolymer chain $\left(q_{A}=q_{B}\right)$ is always localized at the interface.

Since the radius of convergence $K_{*}(\beta)$ and free energy per monomer are linked by equation (四), we get

$$
f(T)=\frac{1}{\beta} \ln \frac{\sqrt{\sinh \left(\beta q_{A}\right) \sinh \left(\beta q_{B}\right)}}{\sinh \beta\left(q_{A}+q_{B}\right)}
$$

for $T \leq T_{c}$, which in turn yields

$$
f(T) \underset{T \rightarrow T_{c}}{\simeq} f_{\text {deloc }}\left(T_{c}\right)-C\left(T_{c}-T\right)^{2}
$$

where $f_{\text {deloc }}\left(T_{c}\right)=-T_{c} \ln 2-q_{0}$ is the free energy of the delocalized phase (since we have chosen $q_{0}>0$, the chain is then in fluid $\alpha$ ). Equation (23) corresponds to a critical exponent $\alpha=0$. The jump in the specific heat per monomer is given by

$$
\begin{aligned}
& C_{\infty}\left(T_{c}^{-}\right)=\left(\frac{\left(2 e^{2 \beta_{c} q_{A}}-1\right) \ln \left(2-e^{-2 \beta_{c} q_{A}}\right)-2 \beta_{c} q_{A}}{2\left(e^{2 \beta_{c} q_{A}}-1\right)}\right)^{2} \\
& C_{\infty}\left(T_{c}^{+}\right)=0
\end{aligned}
$$

\section{Density profile}

We denote the normalized density profile by $\rho(2 n)$ and write

$$
\rho(2 n)=\lim _{N \rightarrow \infty} \frac{Z_{2 N}(2 n)}{Z_{2 N}}
$$


Inverting equations (13) and (14), we have

$$
Z_{2 N}(2 n)=\oint_{\text {circle around } 0} \frac{d K}{2 i \pi} \frac{Z(K, 2 n)}{K^{N+1}}
$$

After deformation of the contour, the dominant contribution for large $N$ comes from the pole at $K_{*}(\beta)$ (see equation (19)), and reads

$$
Z_{2 N}(2 n) \underset{N \text { large }}{\simeq}-\oint_{\text {circle around } K_{*}} \frac{d K}{2 i \pi} \frac{Z(K, 2 n)}{K^{N+1}}
$$

Summing over $n$ leads to

$$
Z_{2 N} \underset{N \text { large }}{\simeq}-\oint_{\text {circle around } K_{*}} \frac{d K}{2 i \pi} \frac{Z(K)}{K^{N+1}}
$$

The density profile $\rho(2 n)$ is then obtained as

$$
\rho(2 n)=\frac{1}{r_{+}\left(K_{*}^{1 / 2} e^{\beta q_{0}}\right)+e^{\beta\left(q_{A}+q_{B}\right)} B\left(K_{*}^{1 / 2} e^{\beta q_{0}}\right) r_{-}\left(K_{*}^{1 / 2} e^{-\beta q_{0}}\right)}\left(\frac{B\left(K_{*}^{1 / 2} e^{\beta q_{0}}\right)}{K_{*}^{1 / 2} e^{\beta q_{0}}}-1\right)^{n}
$$

for $n \geq 1$ and

$$
\rho(2 n)=\frac{e^{\beta\left(q_{A}+q_{B}\right)} B\left(K_{*}^{1 / 2} e^{\beta q_{0}}\right) B\left(K_{*}^{1 / 2} e^{-\beta q_{0}}\right)}{r_{+}\left(K_{*}^{1 / 2} e^{\beta q_{0}}\right)+e^{\beta\left(q_{A}+q_{B}\right)} B\left(K_{*}^{1 / 2} e^{\beta q_{0}}\right) r_{-}\left(K_{*}^{1 / 2} e^{-\beta q_{0}}\right)}\left(\frac{B\left(K_{*}^{1 / 2} e^{-\beta q_{0}}\right)}{K_{*}^{1 / 2} e^{-\beta q_{0}}}-1\right)^{|n|}
$$

for $n \leq 0$. Inserting the value of $K_{*}(\beta)$, we get

$$
\begin{aligned}
& \rho(2 n)=\frac{\left(e^{2 \beta q_{B}}+e^{-2 \beta q_{A}}-2\right)\left(e^{2 \beta q_{A}}+e^{-2 \beta q_{B}}-2\right)}{\left.\left(e^{2 \beta\left(q_{A}+q_{B}\right.}\right)-1\right)\left(1-e^{-2 \beta q_{A}}\right)\left(1-e^{-2 \beta q_{B}}\right)}\left(\frac{1-e^{-2 \beta q_{A}}}{e^{2 \beta q_{B}}-1}\right)^{n} \quad \text { for } n \geq 1 \\
& \rho(2 n)=\frac{\left(e^{2 \beta q_{B}}+e^{-2 \beta q_{A}}-2\right)\left(e^{2 \beta q_{A}}+e^{-2 \beta q_{B}}-2\right)}{\left(e^{2 \beta\left(q_{A}+q_{B}\right)}-1\right)\left(1-e^{-2 \beta q_{A}}\right)\left(1-e^{-2 \beta q_{B}}\right)}\left(\frac{1-e^{-2 \beta q_{B}}}{e^{2 \beta q_{A}}-1}\right)^{-n} \quad \text { for } n \leq 0
\end{aligned}
$$

i.e. the density around the interface decays exponentially from the interface with characteristic lengths $\xi_{\alpha}$ (resp. $\xi_{\beta}$ ) in fluid $\alpha$ (resp. fluid $\beta$ ), with

$$
\begin{aligned}
& \xi_{\alpha}(T)=\frac{2}{\ln \left(\frac{e^{2 \beta q_{B}-1}}{1-e^{-2 \beta q_{A}}}\right)} \\
& \xi_{\beta}(T)=\frac{2}{\ln \left(\frac{e^{2 \beta q_{A}-1}}{1-e^{-2 \beta q_{B}}}\right)}
\end{aligned}
$$

Close to $T_{c}$, we get

$$
\xi_{\alpha}(T) \underset{T \rightarrow T_{c}}{\propto} \frac{1}{T_{c}-T}
$$

and

$$
\xi_{\beta}\left(T_{c}\right)=\frac{T_{c}}{\left(q_{A}+q_{B}\right)}
$$

The order parameter of the transition can be chosen as the probability $\mathcal{M}_{\beta}$ to be in fluid $\beta$ and vanishes linearly at the transition:

$$
\mathcal{M}_{\beta}=\sum_{n=-\infty}^{0} \rho(2 n) \underset{T \rightarrow T_{c}}{\propto}\left(T_{c}-T\right)
$$




\section{E. Probability distribution of loop lengths}

Contrary to the direct matrix transfer approach, the grand canonical approach allows

one to obtain informations on the statistical properties of loops in each fluid. Without loss of generality, we may consider the partition function $Z_{2 N}(2 n=0)$ where the chain of length $2 N$ is attached at the interface at both ends, and decompose it into

$$
Z_{2 N}(2 n=0)=\sum_{l_{1}=1}^{\infty} \sum_{l_{2}=1}^{\infty} Z_{2 N}\left(2 n=0,2 l_{1}-1,2 l_{2}-1\right)
$$

where $Z_{2 N}\left(2 n=0,2 l_{1}-1,2 l_{2}-1\right)$ represents the partition function with the constraints that the first loop in fluid $\alpha$ contains exactly $\left(2 l_{1}-1\right)$ monomers and that the first loop in fluid $\beta$ contains exactly $\left(2 l_{2}-1\right)$ monomers.

We again define generating functions by

$$
\hat{Z}\left(K, s_{1}, s_{2}\right)=\sum_{N=1}^{\infty} \sum_{l_{1}=1}^{\infty} \sum_{l_{2}=1}^{\infty} K^{N} e^{-s_{1}\left(2 l_{1}-1\right)-s_{2}\left(2 l_{2}-1\right)} Z_{2 N}\left(2 n=0,2 l_{1}-1,2 l_{2}-1\right)
$$

The generalization of equation (14) to the case $\left(s_{1}, s_{2}\right) \neq(0,0)$ then yields

$$
\hat{Z}\left(K, s_{1}, s_{2}\right)=\frac{e^{\beta\left(q_{A}+q_{B}\right)} B\left(K^{1 / 2} e^{\beta q_{0}} e^{-s_{1}}\right) B\left(K^{1 / 2} e^{-\beta q_{0}} e^{-s_{2}}\right)}{1-e^{\beta\left(q_{A}+q_{B}\right)} B\left(K^{1 / 2} e^{\beta q_{0}}\right) B\left(K^{1 / 2} e^{-\beta q_{0}}\right)}
$$

Again, by inversion and deformation of the contour in the complex plane, we get the asymptotic behavior for large $N$

$$
\begin{aligned}
& \hat{Z}_{2 N}\left(s_{1}, s_{2}\right)= \oint_{\text {circle around } 0} \frac{d K}{2 i \pi} \frac{\hat{Z}\left(K, s_{1}, s_{2}\right)}{K^{N+1}} \\
& \simeq-\oint_{\text {Nlarge }} \\
& \simeq \text { circle around } K_{*} \frac{d K}{2 i \pi} \frac{\hat{Z}\left(K, s_{1}, s_{2}\right)}{K^{N+1}}
\end{aligned}
$$

The generating functions $\hat{P}_{\alpha}$ and $\hat{P}_{\beta}$ of the loop length distribution functions in the two fluids are given by

$$
\begin{aligned}
& \hat{P}_{\alpha}(s) \equiv \sum_{l_{1}=1}^{\infty} e^{-s\left(2 l_{1}-1\right)} P_{\alpha}\left(2 l_{1}-1\right)=\lim _{N \rightarrow \infty}\left(\frac{\hat{Z}_{2 N}\left(s_{1}=s, s_{2}=0\right)}{\hat{Z}_{2 N}\left(s_{1}=0, s_{2}=0\right)}\right) \\
& \hat{P}_{\beta}(s) \equiv \sum_{l_{2}=1}^{\infty} e^{-s\left(2 l_{2}-1\right)} P_{\beta}\left(2 l_{2}-1\right)=\lim _{N \rightarrow \infty}\left(\frac{\hat{Z}_{2 N}\left(s_{1}=0, s_{2}=s\right)}{\hat{Z}_{2 N}\left(s_{1}=0, s_{2}=0\right)}\right)
\end{aligned}
$$

More explicitely, we have

$$
\begin{aligned}
& \hat{P}_{\alpha}(s)=\frac{B\left(K_{*}^{1 / 2} e^{\beta q_{0}} e^{-s}\right)}{B\left(K_{*}^{1 / 2} e^{\beta q_{0}}\right)}=e^{s} \frac{1-\sqrt{1-e^{-\omega(\beta)-2 s}}}{1-\sqrt{1-e^{-\omega(\beta)}}} \\
& \hat{P}_{\beta}(s)=\frac{B\left(K_{*}^{1 / 2} e^{-\beta q_{0}} e^{-s}\right)}{B\left(K_{*}^{1 / 2} e^{\beta q_{0}}\right)}=e^{s} \frac{1-\sqrt{1-e^{-\omega(\beta)-4 \beta q_{0}-2 s}}}{1-\sqrt{1-e^{-\omega(\beta)-4 \beta q_{0}}}}
\end{aligned}
$$


with

$$
\omega(\beta)=-2 \beta q_{0}-2 \ln 2-\ln K_{*}=2 \beta\left(f_{\text {deloc }}-f\right)=\ln \left(\frac{\left(e^{2 \beta\left(q_{A}+q_{B}\right)}-1\right)^{2}}{4 e^{2 \beta q_{A}}\left(e^{2 \beta q_{A}}-1\right)\left(e^{2 \beta q_{B}}-1\right)}\right)
$$

We note that equations (45) and (46) exhibit several loop size scales, that we consider below.

At the critical temperature $\left(T=T_{c}\right)$, the distribution of loop lengths $\left(l_{\alpha}\right)$ in fluid $\alpha$ is simply the loop distribution of a free random walk

$$
\hat{P}_{\alpha}(s) \underset{T=T_{c}}{=} e^{s}\left[1-\sqrt{1-e^{-2 s}}\right]=1-\sqrt{2 s}+O(s)
$$

yielding an algebraic decay for large $l_{\alpha}$

$$
P_{\alpha}\left(l_{\alpha}\right) \underset{l_{\alpha} \rightarrow \infty}{\simeq} \frac{1}{l_{\alpha}^{3 / 2}}
$$

A consequence of this critical distribution $\left(T=T_{c}\right)$ is as follows. For a chain of length $N$, the number of loops scales as $\sqrt{N}$, the longest $(\alpha)$ loop being of order $N$.

In the critical region $T \rightarrow T_{c}$ where $\omega(\beta) \sim\left(T_{c}-T\right)^{2}$, the moments of $\left(l_{\alpha}\right)$ diverge as

$$
\overline{l_{\alpha}^{n}}=\sum_{l=1}^{\infty}(2 l-1)^{n} P_{\alpha}(2 l-1) \underset{T \rightarrow T_{c}}{\propto}\left(T_{c}-T\right)\left(\frac{1}{\left(T_{c}-T\right)^{2}}\right)^{n}
$$

i.e. for large $l_{\alpha}$ the correct scaling variable is $\lambda=l_{\alpha}\left(T_{c}-T\right)^{2}$, but the normalisation of the region of finite $\lambda$ varies as $\left(T_{c}-T\right)$. This can be understood by considering the normalisation of the region of loops bigger than a given scale $l_{c} \sim \frac{1}{\left(T_{c}-T\right)^{2}}$ for a free random walk (49)

$$
\int_{l_{c}}^{\infty} d l \frac{1}{l^{3 / 2}} \sim \frac{1}{l_{c}^{1 / 2}} \sim\left(T_{c}-T\right)
$$

In other words, the phase transition is driven by a small fraction of large loops.

It is of interest to note that the weight of the small $l_{\alpha}$ loops is finite, even at $T_{c}$. For instance, the probability to have $l_{\alpha}=1$ is

$$
P_{\alpha}\left(l_{\alpha}=1\right)=\frac{e^{2 \beta q_{B}}-1}{e^{2 \beta q_{B}}-e^{-2 \beta q_{A}}} \underset{T \rightarrow T_{c}}{\simeq} \frac{1}{2}
$$

At zero temperature we of course recover the ground state

$$
P_{\alpha}\left(l_{\alpha}\right) \underset{T=0}{\simeq} \delta_{l_{\alpha}, 1}
$$

In marked contrast, the critical distribution of loop lengths in the $(\beta)$ fluid is given by

$$
\hat{P}_{\beta}(s) \underset{T=T_{c}}{\simeq} e^{s} \frac{1-\sqrt{1-e^{-4 \beta_{c} q_{0}-2 s}}}{1-\sqrt{1-e^{-4 \beta_{c} q_{0}}}}
$$

leading to finite $\overline{l_{\beta}^{n}}$ moments. 


\section{F. Correlation function}

To further emphasize the meaning of the scale corresponding to $\omega(\beta)$ in equation (47), we consider the connected correlation function

$$
\mathcal{C}(j-i)=<\operatorname{sgn}\left[z(i)-\frac{1}{2}\right] \operatorname{sgn}\left[z(j)-\frac{1}{2}\right]>-<\operatorname{sgn}\left[z(i)-\frac{1}{2}\right]><\operatorname{sgn}\left[z(j)-\frac{1}{2}\right]>
$$

Its generating function reads

$$
\hat{\mathcal{C}}(K)=\sum_{j=i}^{\infty} K^{j-i} \mathcal{C}(j-i)
$$

and can be expressed, via a loop decomposition, in terms of the functions $\hat{P}_{\alpha}(s)$ and $\hat{P}_{\beta}(s)$ defined in (45,46), with the replacement $(s=-\ln K)$. Inverting (56), we have

$$
\mathcal{C}(j-i)=\oint_{\text {circle around } 0} \frac{d K}{2 i \pi} \frac{\hat{\mathcal{C}}(K)}{K^{1+j-i}}
$$

At large $(j-i)$ separation, the behaviour of $\mathcal{C}(j-i)$ is dominated by the singularity at $K=e^{\frac{\omega(\beta)}{2}}$ of $\hat{P}_{\alpha}(-\ln K)$, leading to

$$
\mathcal{C}(j-i) \underset{(j-i) \rightarrow \infty}{\propto} e^{-(j-i) \frac{\omega(\beta)}{2}}
$$

This shows that $\omega(\beta)$ is the inverse of the correlation length along the chain.

\section{G. Finite size properties}

In this section, we again consider that both chain ends are fixed at the interface. This means in particular that one has $Z_{2 N}=Z_{2 N}(0), Z(K)=Z(K, 0), \ldots$.

\section{Free energy}

In the inversion formula of equation (27), we set $2 n=0$ and get

$$
Z_{2 N}(0)=\oint_{\text {circle around } 0} \frac{d K}{2 i \pi} \frac{Z(K, 0)}{K^{N+1}}
$$

where $Z(K, 0)$ given in (14) presents a simple pole at $K_{*}(\beta)$, and a cut on the real axis, namely $\left[K_{\text {deloc }}(\beta),+\infty\right]$.

In the localized phase, and after deformation of the contour, the dominant contribution for large $N$ comes from the pole at $K_{*}(\beta)$, and the correction is given by the leading contribution of the cuts. Simplifying as explained above the notations, we have

$$
\begin{aligned}
Z_{2 N} \underset{\text { Nlarge }}{\simeq}-\oint_{\text {circle around } K_{*}} \frac{d K}{2 i \pi} \frac{Z(K)}{K^{N+1}}+O\left(\frac{1}{K_{\text {deloc }}^{N}}\right) \\
\underset{\text { Nlarge }}{\simeq} \frac{1}{K_{*}^{N}}\left(\rho(0)+O\left(\frac{K_{*}}{K_{\text {deloc }}}\right)^{N}\right)
\end{aligned}
$$


where $\rho(0)$ given in (33) represents the probability to be at $2 n=0$.

In the delocalized phase, the finite size properties are given by the contributions of the cuts

$$
Z_{2 N}=\int_{0}^{+\infty} \frac{d x}{2 i \pi} \frac{1}{K_{\text {deloc }}^{N}(1+x)^{N+1}}\left[Z\left(K_{\text {deloc }}(1+x+i o)\right)-Z\left(K_{\text {deloc }}(1+x-i o)\right)\right]
$$

To obtain the dominant behavior at large $N$, we will set $v=N \ln (1+x)$.

For $T>T_{c}$, using

$$
Z\left(K_{\text {deloc }}(1+x+i o)\right)-Z\left(K_{\text {deloc }}(1+x-i o)\right)=\frac{2 i \sqrt{x}(1+G(\beta))}{G(\beta)^{2}}(1+O(x))
$$

with

$$
G(\beta)=e^{2 \beta q_{A}}\left(1-\sqrt{1-e^{-4 \beta q_{0}}}\right)-1
$$

we get the asymptotic behavior

$$
\begin{aligned}
& Z_{2 N} \underset{\text { Nlarge }}{\simeq} \int_{0}^{+\infty} \frac{d v}{2 N i \pi} \frac{1}{K_{\text {deloc }}^{N}} e^{-v}\left[Z\left(K_{\text {deloc }}\left(e^{\frac{v}{N}}+i o\right)\right)-Z\left(K_{\text {deloc }}\left(e^{\frac{v}{N}}-i o\right)\right)\right] \\
& \underset{\text { Nlarge }}{\simeq} \frac{1}{K_{\text {deloc }}^{N} \sqrt{\pi} N^{3 / 2}} \frac{(1+G(\beta))}{2 G(\beta)^{2}}
\end{aligned}
$$

For $T=T_{c}, G\left(\beta_{c}\right)$ vanishes and we have

$$
Z\left(K_{\text {deloc }}(1+x+i o)\right)-Z\left(K_{\text {deloc }}(1+x-i o)\right)=i \frac{2}{\sqrt{x}}(1+O(x))
$$

leading to

$$
\begin{aligned}
Z_{2 N} & \simeq \underset{\text { large }}{\simeq} \int_{0}^{+\infty} \frac{d v}{2 N i \pi} \frac{1}{K_{\text {deloc }}^{N}} e^{-v}\left[Z\left(K_{\text {deloc }}\left(e^{\frac{v}{N}}+i o\right)\right)-Z\left(K_{\text {deloc }}\left(e^{\frac{v}{N}}-i o\right)\right)\right] \\
& \simeq \frac{1}{N \operatorname{large}} \frac{1}{K_{\text {deloc }}^{N} \sqrt{\pi N}}
\end{aligned}
$$

This shows that the probability of presence at the interface decays with the length $N$ as $1 / N^{1 / 2}$ at $T=T_{c}(69)$, whereas it decays as $1 / N^{3 / 2}$ for $T>T_{c}$.

In the following, we will need the general scaling form which contains (66) and (69) as special cases

$$
Z_{2 N} \underset{\text { Nlarge }}{\simeq} \frac{1+G(\beta)}{K_{\text {deloc }}^{N} \sqrt{N} H(\beta)} q\left(\sqrt{N} \frac{G(\beta)}{\sqrt{H(\beta)}}\right)
$$

where the scaling function $q(x)$ reads

$$
q(x)=\frac{1}{\pi} \int_{0}^{+\infty} d v \frac{e^{-v} \sqrt{v}}{v+x^{2}}
$$


and

$$
H(\beta)=\frac{e^{4 \beta q_{B}}\left(1-e^{-2 \beta q_{A}}\right)}{\sqrt{1-e^{-4 \beta q_{0}}}\left(1+\sqrt{1-e^{-4 \beta q_{0}}}\right)^{2}}
$$

and where $G(\beta)$ has been defined in (64).

In particular, near $T_{c}$, we have for $T \geq T_{c}$ the scaling form

$$
Z_{2 N} \underset{\text { Nlarge }, T \rightarrow T_{c}^{+}}{\simeq} \frac{1}{K_{\text {deloc }}^{N} \sqrt{N}} q\left(\frac{\left(T-T_{c}\right)}{T_{c}} \sqrt{N C_{\infty}\left(T_{c}^{-}\right)}\right)
$$

leading to the finite size free-energy

$$
f_{2 N}=\frac{-T \ln Z_{2 N}}{2 N} \underset{N \operatorname{large}, T \rightarrow T_{c}^{+}}{\simeq} f_{\text {deloc }}\left(T_{c}\right)+\frac{T_{c}}{4 N} \ln N-\frac{T_{c}}{2 N} \ln q\left(\frac{\left(T-T_{c}\right)}{T_{c}} \sqrt{N C_{\infty}^{-}\left(T_{c}\right)}\right)
$$

\section{Specific heat}

In the thermodynamic limit, the specific heat $C_{\infty}(T)=-T \frac{d^{2} f(T)}{d T^{2}}$ presents a jump at $T_{c}$ given in equation (24). To compute the finite-size specific heat $C_{2 N}\left(T_{c}\right)$ at $T=T_{c}$, we use the scaling form (74)

$$
\begin{aligned}
C_{2 N}\left(T_{c}\right) & =-\left.T \frac{d^{2} f_{2 N}}{d T^{2}}\right|_{T=T_{c}}=\frac{1}{2}\left(\frac{q^{\prime \prime}(0)}{q(0)}-\left(\frac{q^{\prime}(0)}{q(0)}\right)^{2}\right) C_{\infty}\left(T_{c}^{-}\right)+\frac{q^{\prime}(0)}{q(0)} \frac{\sqrt{C_{\infty}\left(T_{c}^{-}\right)}}{\sqrt{N}}+. . \\
& =\frac{4-\pi}{2} C_{\infty}\left(T_{c}^{-}\right)-\sqrt{\pi} \frac{\sqrt{C_{\infty}\left(T_{c}^{-}\right)}}{\sqrt{N}}+. .
\end{aligned}
$$

Similar relations between $C_{2}\left(T_{c}\right)$ and $C_{\infty}\left(T_{c}\right)$ are found in phase transitions displaying a jump in the specific heat 16.17.

\section{Order parameter and uniform susceptibility}

A possible order parameter at the transition has been mentionned in equation (38), and linked to the probability to be in fluid $\beta$. We consider here

$$
M_{2 N}=\sum_{i=1}^{2 N} \operatorname{sgn}\left[z(i)-\frac{1}{2}\right]
$$

and the order parameter $m_{2 N}=\frac{\leq M_{2 N}>}{2 N}$. Note that $m_{2 N}$ is related to the quantity $\mathcal{M}_{\beta}$ of (38) by $\left(m_{2 N}=1-2 \mathcal{M}_{\beta}\right)$. Given the expression of the Hamiltonian (11), we get

$$
\begin{aligned}
m_{\infty}=<\operatorname{sgn}\left[z(i)-\frac{1}{2}\right]> & =-\left.\partial_{h} f\left(T, q_{A} \rightarrow q_{A}+h, q_{B} \rightarrow q_{B}-h\right)\right|_{h=0} \\
& =\frac{\sinh \beta\left(q_{A}-q_{B}\right)}{2 \sinh \beta q_{A} \sinh \beta q_{B}} \underset{T \rightarrow T_{c}^{-}}{\simeq} 1-\operatorname{Cte}\left(T_{c}-T\right)+. .
\end{aligned}
$$


In the thermodynamic limit, the corresponding susceptibility reads

$$
\begin{aligned}
\chi_{\infty}=\frac{1}{2 T N}\left(<M_{2 N}^{2}>-\left(<M_{2 N}>\right)^{2}\right) & =-\left.\partial_{h}^{2} f\left(T, q_{A} \rightarrow q_{A}+h, q_{B} \rightarrow q_{B}-h\right)\right|_{h=0} \\
& =\frac{1}{2 T}\left(\frac{1}{\sinh ^{2} \beta q_{A}}+\frac{1}{\sinh ^{2} \beta q_{B}}\right)
\end{aligned}
$$

leading, at criticality, to

$$
\chi_{\infty}\left(T_{c}^{-}\right)=\frac{4 \beta_{c}}{\left(1-e^{-2 \beta_{c} q_{A}}\right)^{2}}
$$

To compute the finite-size behavior of the order parameter $m_{2 N}$ and of the susceptibility $\chi_{2 N}$, we use again the scaling form (74), but where now $T_{c}$ is a function of $h$ defined by the equation for $T_{c}$ where $q_{A} \rightarrow q_{A}+h, q_{B} \rightarrow q_{B}-h$. Since the calculations exactly parallel the ones described above, we only quote the results

$$
m_{2 N}\left(T_{c}\right)=1-\frac{\sqrt{2 \pi}}{\left(1-e^{-2 \beta_{c} q_{A}}\right)} \frac{1}{\sqrt{2 N}} \ldots
$$

and

$$
\chi_{2 N}\left(T_{c}\right)=\frac{4-\pi}{2} \chi_{\infty}\left(T_{c}^{-}\right)
$$

\section{Numerical results}

We have done numerical calculations using transfer matrix methods with both chain ends fixed at the interface. We chose $q_{A}=1$ and $q_{B}=0.5$, yielding a critical temperature $T_{c}=1 /\left(\ln \left(\frac{1+\sqrt{5}}{2}\right)\right)=2.07809 \ldots$ (see equation $\left.(20)\right)$. The corresponding values of the critical specific heat and susceptibility are $C_{\infty}\left(T_{c}^{-}\right)=0.11056 \ldots$ and $\chi_{\infty}\left(T_{c}^{-}\right)=5.03932 \ldots$ (see equations (24), (80)).

Results for $C_{2 N}(T)$ and $\chi_{2 N}(T)$ in the critical region are shown in Figures 1 and 2, for chain length up to $2 N=18000$ and are in quantitative agreement with the above values. We get for instance a value $C_{2 N}\left(T_{c}\right) \simeq 0.04739$.. as compared to the value $0.047454 \ldots$ coming from equation $(\sqrt{76})$, and $\chi_{2 N}\left(T_{c}\right) \simeq 2.13376 \ldots$ as compared to the value $2.16289 \ldots$ coming

from equation (82). We have also tested equation (81): Figure 3 shows the behaviour of $\sqrt{2 N} \frac{\left(1-m_{2 N}(T)\right)}{2}$ in the critical region. At $T_{c}$, the theoretical value coming from equation (81) is $2.0279 \ldots$ as compared to the "experimental" value 2.0130.. of Figure 3. 


\section{THE WETTING TRANSITION}

\section{A. Calculation of the grand canonical partition function}

We consider the SOS version of the 2D wetting problem near a attracting wall at $h=0$. Our presentation will be rather brief, and we follow the notations of $\mathrm{B}^{\mathrm{a}}$ and . Let us denote by $\{h(i), i=1,2, . ., N\}$ the $N$ consecutive heigths with the properties $h(i)=0,1,2 \ldots$ and $h(i+1)-h(i)=+1,0,-1$. This height model can also be considered as describing the adsorption of a polymer chain onto the wall. We further assume that the first height is fixed $(h(i=1)=1)$. The partition function of the model reads

$$
Z_{N}=\sum_{\{h(i)\}} \exp \left(-\beta J \sum_{i=1}^{N-1}|h(i+1)-h(i)|+\beta u_{0} \sum_{i=1}^{N} \delta_{h_{i}, 0}\right)
$$

Following closely the steps of the previous section, we first express the partition function $Z_{N}$ as a function of its end-point $(h(N)=h)$, and write

$$
Z_{N}=\sum_{h=0}^{+\infty} Z_{N}(h)
$$

At this stage, it is convenient to introduce some notations and relations.

Let $\mathcal{B}(l)$ represent the partition function for a chain starting from $h=1$ and arriving at $h=0$ for the first time after $l$ steps. We then denote by $\mathcal{M}(l-1, h)$ the partition function for a chain of $(l-1)$ steps going from $h=1$ to $h \geq 1$ in the presence of an absorbing boundary at $h=0$. Setting $t=e^{-\beta J}$, the method of images 14 yields

$$
\mathcal{B}(l)=t(R(l-1,0)-R(l-1,2))
$$

and

$$
\mathcal{M}(l-1, h)=R(l-1, h-1)-R(l-1, h+1)
$$

where $R(l, h)$ denotes the partition function of the chain in the absence of the wall. We explicitly have

$$
R(l, h)=\sum_{p=|h|}^{l} C_{l}^{p} C_{p}^{\frac{p+|h|}{2}} t^{p}
$$

normalized to $\sum_{h=-l}^{+l} R(l, h)=(1+2 t)^{l}$.

We now decompose $Z_{N}(h)$ into free and adsorbed segments as

$$
\begin{aligned}
Z_{N}(h)= & \sum_{p=0}^{+\infty} \sum_{l_{1}=1}^{\infty} \sum_{l_{2}=1}^{\infty} \ldots \sum_{l_{2 p}=1}^{\infty} \sum_{l=1}^{\infty} \delta\left(N-\left(\sum_{i=1}^{2 p} l_{i}+l\right)\right) \\
& \mathcal{B}\left(l_{1}\right)\left(e^{\beta u_{0} l_{2}} t\right) \mathcal{B}\left(l_{3}\right)\left(e^{\beta u_{0} l_{4}} t\right) \ldots\left(e^{\beta u_{0} l_{2 p}} t\right) \mathcal{M}(l-1, h) \quad \text { for } h \geq 1 \\
Z_{N}(0)= & \sum_{p=0}^{+\infty} \sum_{l_{1}=1}^{\infty} \sum_{l_{2}=1}^{\infty} \ldots \sum_{l_{2 p}=1}^{\infty} \delta\left(N-\left(\sum_{i=1}^{2 p} l_{i}+l\right)\right) \mathcal{B}\left(l_{1}\right)\left(e^{\beta u_{0} l_{2}} t\right) \ldots \mathcal{B}\left(l_{2 p-1}\right) e^{\beta u_{0} l_{2 p}}
\end{aligned}
$$


It is again convenient to consider the grand canonical partition function

$$
Z(K)=\sum_{N=1}^{+\infty} K^{N} Z_{N}
$$

Using

$$
\hat{\mathcal{B}}(K)=\sum_{l=1}^{+\infty} K^{l} \mathcal{B}(l)=\frac{1-\sqrt{1-4\left(\frac{t K}{1-K}\right)^{2}}}{2 \frac{t K}{1-K}}
$$

we finally have

$$
\begin{gathered}
Z(K, 2 h)=\frac{1}{1-\hat{\mathcal{B}}(K) \frac{t K e^{\beta u_{0}}}{1-K e^{\beta u_{0}}}} \frac{1}{t}\left(\frac{1-K}{t K} \hat{\mathcal{B}}(K)-1\right)^{h} \\
Z(K, 2 h+1)=\frac{1}{1-\hat{\mathcal{B}}(K) \frac{t K e^{\beta u_{0}}}{1-K e^{\beta u_{0}}}} \frac{1}{t} \hat{\mathcal{B}}(K)\left(\frac{1-K}{t K} \hat{\mathcal{B}}(K)-1\right)^{h}
\end{gathered}
$$

\section{B. Thermodynamics}

Equations (92) and (93) give

$$
Z(K)=\sum_{h=0}^{+\infty} Z(K, h)=\frac{1}{1-\hat{\mathcal{B}}(K) \frac{t K e^{\beta u_{0}}}{1-K e^{\beta u_{0}}}} \frac{1+\hat{\mathcal{B}}(K)}{t} \frac{1}{2-\frac{1-K}{t K} \hat{\mathcal{B}}(K)}
$$

The radius of convergence $K_{*}(\beta)$ of $Z(K)$ is either given by the solution of the equation $\left(2-\frac{1-K}{t K} \hat{\mathcal{B}}(K)=0\right)$, or by the solution of the equation $\left(1-\hat{\mathcal{B}}(K) \frac{t K e^{\beta u_{0}}}{1-K e^{\beta u_{0}}}=0\right)$. The former corresponds to a value

$$
K_{\text {deloc }}=\frac{1}{1+2 t}
$$

and physically describes the delocalized phase. The latter is given by

$$
K_{*}(\beta)=\frac{2 e^{-\beta u_{0}}}{1+\sqrt{\frac{e^{\beta u_{0}-1+4 t^{2}}}{e^{\beta u} u_{0}-1}}}
$$

The chain is localized close to the wall as long as $K_{*}(\beta)<K_{\text {deloc }}$, i.e. $T<T_{c}=1 / \beta_{c}$ with

$$
e^{\beta_{c} u_{0}}=\frac{1+2 t}{1+t}
$$

in agreement with references 3 . 


\section{Density profile}

We follow equation (26) and define a normalized density profile by

$$
\rho(h)=\lim _{N \rightarrow \infty} \frac{Z_{N}(h)}{Z_{N}}
$$

Inverting equation 92, we get

$$
\rho(h)=\rho(0)\left[\frac{1}{2 t}\left(\sqrt{\frac{e^{\beta u_{0}}-1+4 t^{2}}{e^{\beta u_{0}}-1}}-1\right)\right]^{h}
$$

in the localized phase. The characteristic length reads

$$
\xi(T)=-\frac{1}{\ln \left[\frac{1}{2 t}\left(\sqrt{\frac{e^{\beta u_{0}}-1+4 t^{2}}{e^{\beta u} 0}}-1\right)\right]}
$$

and diverges at the transition as

$$
\xi(T) \underset{T \rightarrow T_{c}}{\propto} \frac{1}{T_{c}-T}
$$

in agreement with equation (7.8) of reference 15 . The fraction of adsorbed monomers vanishes as

$$
\rho(h=0) \underset{T \rightarrow T_{c}}{\propto} T_{c}-T
$$

\section{Probability distributions of adsorbed and desorbed segments}

Since the detailed procedure has been given in section [1E, our presentation will be rather sketchy, and we only give the main results. Let us denote by A (resp. D) the adsorbed (resp. desorbed) monomers of the chain. The probability distribution of desorbed loop lengths will be denoted by $P_{D}(l)$, and its Laplace transform by $\hat{P_{D}}(s)$. We get

$$
\hat{P_{D}}(s)=\sum_{l=1}^{\infty} e^{-s l} P_{D}(l)=\frac{\hat{\mathcal{B}}\left(K_{*} e^{-s}\right)}{\hat{\mathcal{B}}\left(K_{*}\right)}
$$

yielding, at criticality, to

$$
\hat{P_{D}}(s) \underset{T=T_{c}}{=} \hat{\mathcal{B}}\left(\frac{1}{1+2 t} e^{-s}\right)=1-\sqrt{\frac{1+2 t}{t}} \sqrt{s}+O(s)
$$

This corresponds to an algebraic decay of $P_{D}(l)$ for large $(l)$, as in equation (49). Similarly, in the region $T \rightarrow T_{c}$ the moments of $P_{D}(l)$ diverge as

$$
\sum_{l=1}^{\infty} l^{n} P_{D}(l) \underset{T \rightarrow T_{c}}{\propto}\left(T_{c}-T\right)\left(\frac{1}{\left(T_{c}-T\right)^{2}}\right)^{n}
$$


As in the copolymer case, the correlation length along the chain scales as $\left(\frac{1}{\left(T_{c}-T\right)^{2}}\right)$, in agreement with equation (6.22) of reference $\frac{15}{5}$. As previously found, the small loops have a finite weight at $T_{c}$. One has for instance

$$
P_{D}(l=1) \underset{T=T_{c}}{=} \frac{t}{1+2 t}
$$

As for the probability distribution of the adsorbed segment lengths, we find an exponential form, even at $T_{c}$.

$$
P_{A}(l)=\left(1-K_{*} e^{\beta u_{0}}\right)\left(K_{*} e^{\beta u_{0}}\right)^{l-1}
$$

where $K_{*}$ is given in equation (96).

We conclude this section on wetting by pointing out that a finite size relation for the specific heat, quite similar to the one derived in equation (76), has been found in referencel

\section{CONCLUSION}

We have presented a grand canonical approach to the localization of a single polymer chain either at a fluid-fluid interface, or at an attracting hard wall. This method, which rests on the absence of self avoidance in the models, provides detailed informations on the loop length distribution function. The extension to various disordered situations of great interest. 


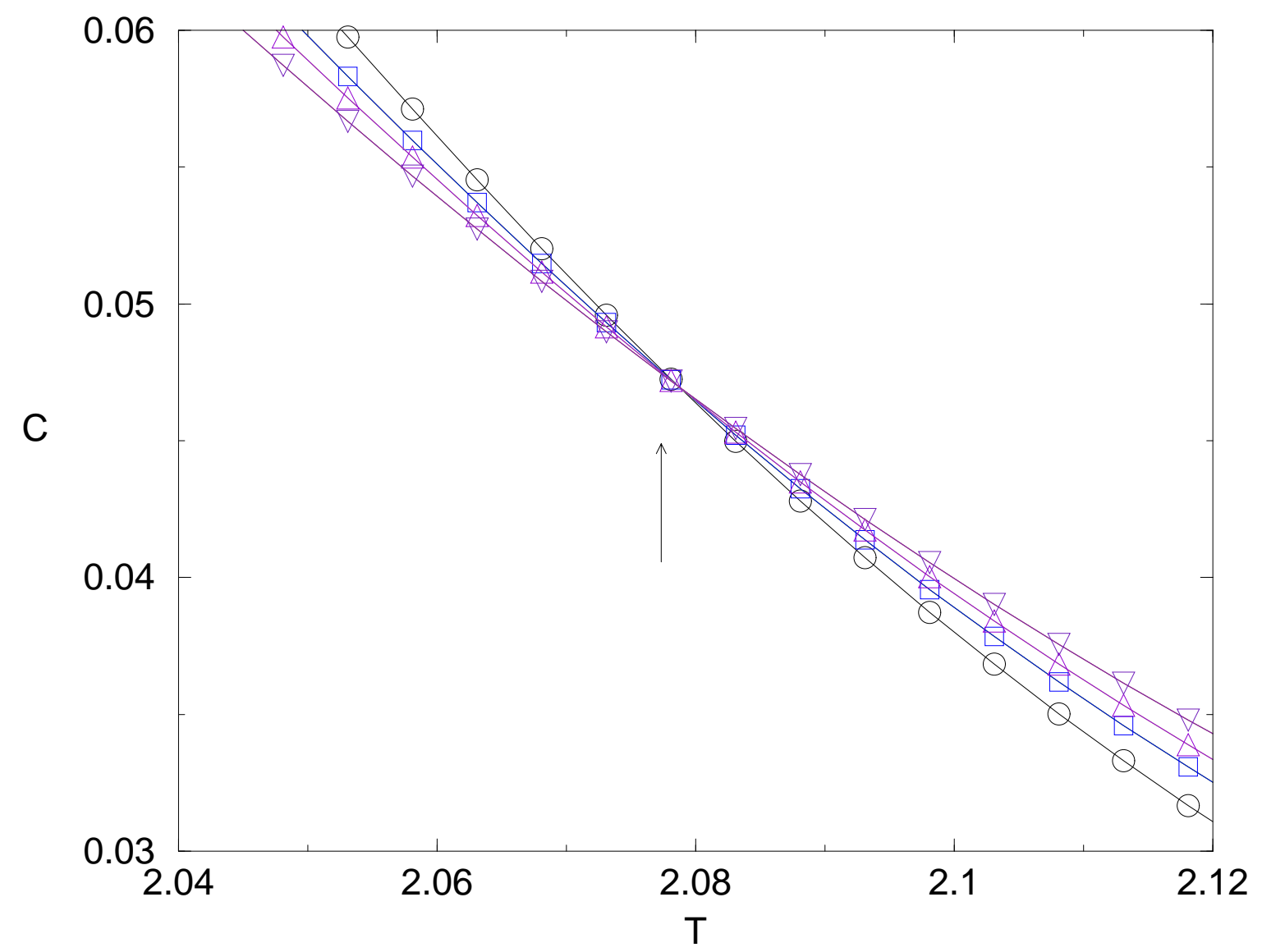

FIG. 1. Specific heat per monomer vs temperature for $q_{A}=1$ and $q_{B}=0.5$ in the critical region for $2 N=18000(\circ), 14000(\square), 12000(\triangle), 10000(\nabla)$. The arrow denotes the critical temperature $T_{c}=2.078 \ldots$, where equation $(76)$ predicts $C\left(T_{c}\right)=0.047454 \ldots$. 


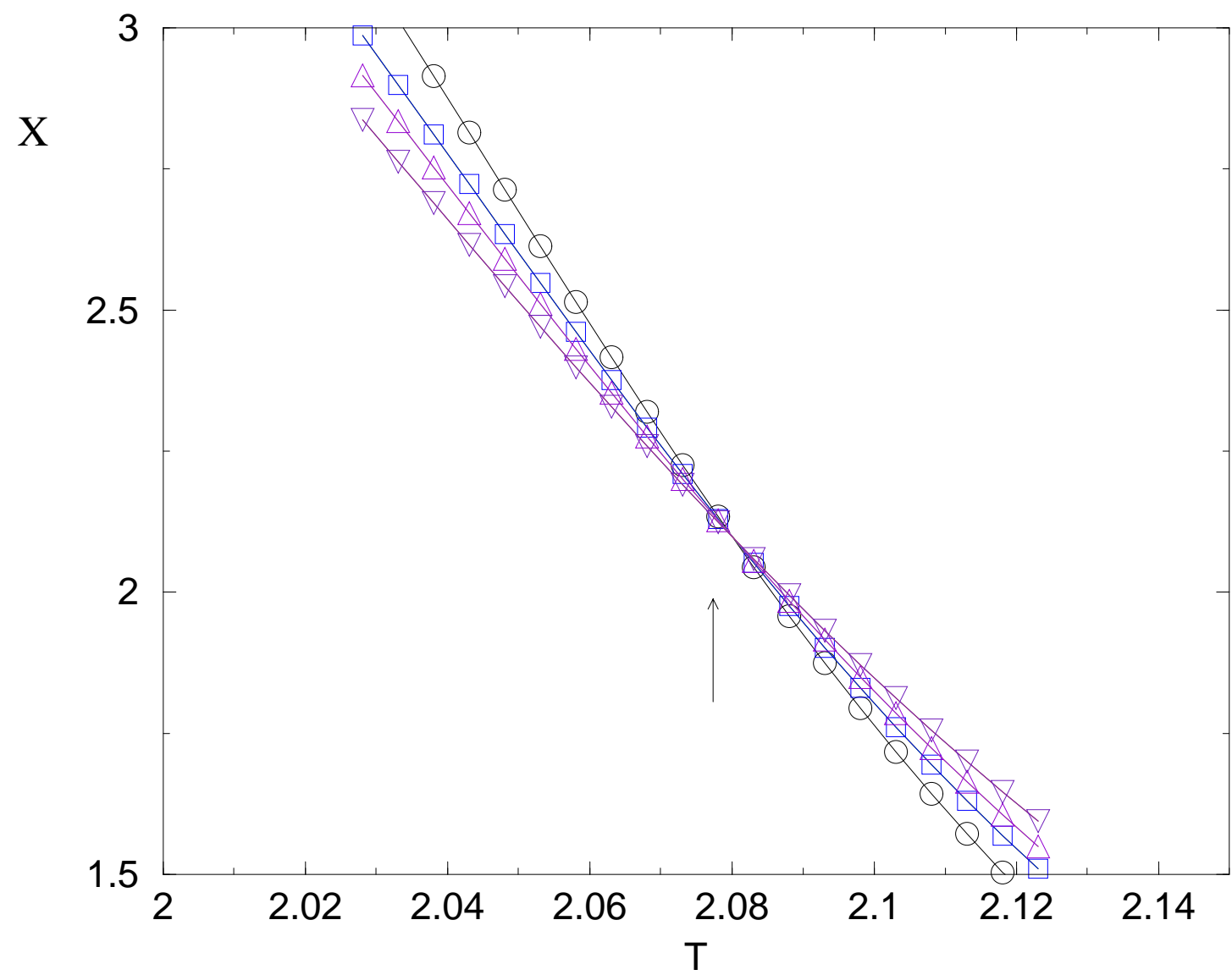

FIG. 2. Susceptibility per monomer vs temperature for $q_{A}=1$ and $q_{B}=0.5$ in the critical region for $2 N=18000(\circ), 14000(\square), 12000(\triangle), 10000(\nabla)$. The arrow denotes the critical temperature $T_{c}=2.078 \ldots$, where equation (82) predicts $\chi\left(T_{c}\right)=2.16289 \ldots$ 


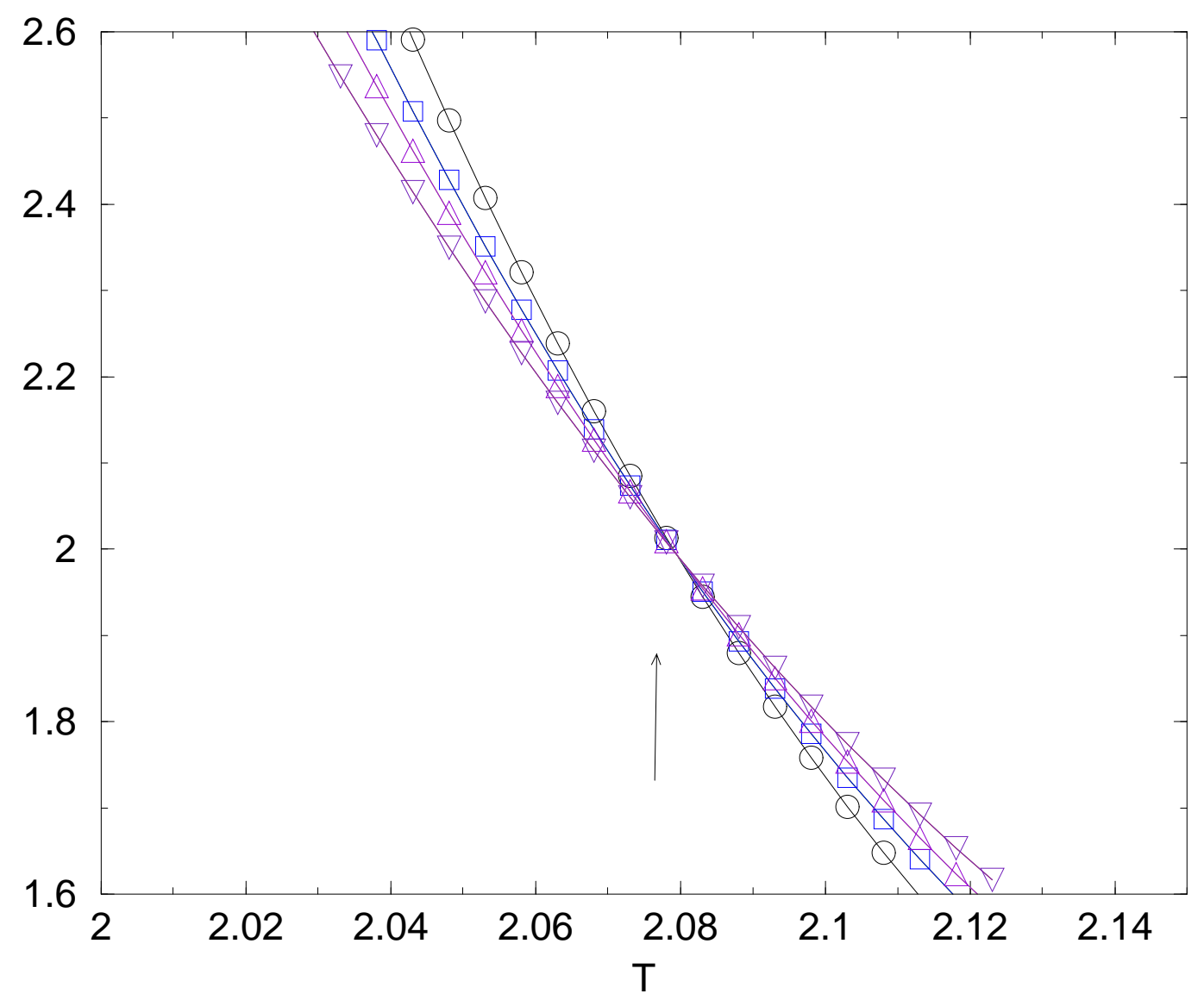

FIG. 3. Test of equation (81): Plot of $\sqrt{2 N} \frac{\left(1-m_{2 N}(T)\right)}{2}$ vs temperature for $q_{A}=1$ and $q_{B}=0.5$ for $2 N=18000(\circ), 14000(\square), 12000(\triangle), 10000(\nabla)$. The arrow denotes the critical temperature $T_{c}=2.078 \ldots$, where equation (81) predicts a value 2.0279. . 


\section{APPENDIX A: TRANSFER MATRIX APPROACH}

In this appendix, we will briefly present the transfer matrix calculations for the periodic $A B$ copolymer chain. We decompose $Z_{N}$ according to the position $n$ of the end point of the chain, $\left(Z_{N}=\sum_{n=-\infty}^{+\infty} Z_{N}(n)\right)$, and using the Hamiltonian of equation (1), we obtain the recursion relations

$$
Z_{N+1}(n)=e^{\beta q_{N+1} \operatorname{sgn}\left[n-\frac{1}{2}\right]}\left(Z_{N}(n+1)+Z_{N}(n-1)\right)
$$

It is convenient to introduce the Fourier transforms for the two half-spaces

$$
\begin{aligned}
& \hat{Z}_{N}^{+}(k)=\sum_{n=1}^{+\infty} e^{i k(n-1)} Z_{N}(n) \\
& \hat{Z}_{N}^{-}(k)=\sum_{n=-\infty}^{0} e^{i k n} Z_{N}(n)
\end{aligned}
$$

as well as their inverse

$$
\begin{aligned}
& Z_{N}(n)=\int_{0}^{2 \pi} \frac{d k}{2 \pi} e^{-i k(n-1)} \hat{Z}_{N}^{+}(k) \quad \text { for } n \geq 1 \\
& Z_{N}(n)=\int_{0}^{2 \pi} \frac{d k}{2 \pi} e^{-i k n} \hat{Z}_{N}^{-}(k) \quad \text { for } \quad n \leq 0
\end{aligned}
$$

The recursion relations now read

$$
\begin{aligned}
& \hat{Z}_{N+1}^{+}(k)=e^{\beta q_{N+1}}\left(2 \cos k \hat{Z}_{N}^{+}(k)-e^{-i k} Z_{N}(1)+Z_{N}(0)\right) \\
& \hat{Z}_{N+1}^{-}(k)=e^{-\beta q_{N+1}}\left(2 \cos k \hat{Z}_{N}^{-}(k)-e^{i k} Z_{N}(0)-Z_{N}(1)\right)
\end{aligned}
$$

To study the thermodynamic limit for our problem, $\left(q_{2 p+1}=q_{A}>0\right.$ and $\left.q_{2 p}=-q_{B}<0\right)$, we look for a stationnary solution $\hat{Z}_{2 N}^{ \pm}(k)$ with eigenvalue $\lambda$. We find

$$
\begin{aligned}
& \hat{Z}^{+}(k)=\frac{1}{\lambda e^{\beta\left(q_{B}-q_{A}\right)}-4 \cos ^{2} k}\left[-e^{-i k} Z(2)+e^{i k} Z(0)\right] \\
& \hat{Z}^{-}(k)=\frac{1}{\lambda e^{-\beta\left(q_{B}-q_{A}\right)}-4 \cos ^{2} k}\left[\left(e^{2 \beta q_{A}}-1-e^{i 2 k}\right) Z(0)+e^{2 \beta q_{A}} Z(2)\right]
\end{aligned}
$$

Using the inversion relations (A5), we obtain the following consistency equations for $Z(2)$ and $Z(0)$

$$
\begin{aligned}
& Z(2)=-I_{2}^{+}(\lambda) Z(2)+I_{0}^{+}(\lambda) Z(0) \\
& Z(0)=e^{2 \beta q_{A}} I_{0}^{-}(\lambda) Z(2)+\left(\left(e^{2 \beta q_{A}}-1\right) I_{0}^{-}(\lambda)-I_{2}^{-}(\lambda)\right) Z(0)
\end{aligned}
$$

whith the notations

$$
\begin{aligned}
& I_{p}^{ \pm}(\lambda)=\frac{1}{4} J_{p}\left(y=\frac{\lambda e^{ \pm \beta\left(q_{B}-q_{A}\right)}}{4}\right) \\
& J_{0}(y)=\int_{-\pi}^{+\pi} \frac{d k}{2 \pi} \frac{1}{y-\cos ^{2} k}=\frac{1}{\sqrt{y(y-1)}} \\
& J_{2}(y)=\int_{-\pi}^{+\pi} \frac{d k}{2 \pi} \frac{\cos 2 k}{y-\cos ^{2} k}=2 \sqrt{\frac{y}{y-1}}-2-\frac{1}{\sqrt{y(y-1)}}
\end{aligned}
$$


For a localized eigenstate, both $Z(2)$ and $Z(0)$ are non zero. As a consequence, the determinant of the system ( $\mathrm{A} 10$ ) has to vanish, and this gives an equation for the eigenvalue $\lambda$ of the localized state, which reads in our case $\left(q_{A} \geq q_{B}\right)$

$$
\lambda=\frac{1}{K_{*}(\beta)}
$$

where $K_{*}(\beta)$ is given in equation (19). The free energy is then given by

$$
f(T)=\lim _{N \rightarrow \infty} \frac{-\ln Z_{2 N}}{\beta 2 N}=-\frac{\ln \lambda}{2 \beta}
$$

in agreement with equation (㺼).

As a final remark on this approach, we mention how one may find the polymer density profile $\rho(2 n)$ in the localized phase. The Fourier transforms of $\rho(2 n)$ on the two-half spaces are indeed directly related to the localized eigenvector $\left(\hat{Z}^{+}(k)\right)$ given in (A8) by

$$
\begin{aligned}
& \sum_{n=0}^{+\infty} e^{i k(2 n-1)} \rho(2 n)=\frac{\hat{Z}^{+}(k)}{\hat{Z}^{+}(0)+\hat{Z}^{-}(0)} \\
& \sum_{n=-\infty}^{0} e^{i k 2 n} \rho(2 n)=\frac{\hat{Z}^{-}(k)}{\hat{Z}^{+}(0)+\hat{Z}^{-}(0)}
\end{aligned}
$$

in agreement with the result (33). All the results of section II can be thus recovered, except for the results of the loop length distribution function of section $\amalg \mathrm{E}$. 


\section{REFERENCES}

${ }^{1}$ G.J. Fleer, M.A. Cohen Stuart, J.M. Scheutjens, T. Cosgrove and B. Vincent, Polymers at interfaces, Chapman and Hall, London (1993).

${ }^{2}$ K. De'Bell and Turab Lookman, Reviews of Mod. Phys., 65, 87 (1993).

${ }^{3}$ G. Forgacs, J.M. Luck, T.M. Nieuwenhuizen and H. Orland, Phys. Rev. Lett., 57, 2184 (1986).

${ }^{4}$ B. Derrida, V. Hakim and J. Vannimenus, J. Stat. Phys., 66, 1189 (1992).

${ }^{5}$ A. Grosberg, S. Izrailev and S. Nechaev, Phys. Rev. E, 50, 1912 (1994).

${ }^{6}$ S. Nechaev and Y-C. Zhang, Phys. Rev. Lett., 74, 1815 (1995).

${ }^{7}$ J.U. Sommer and M. Daoud, Europhys. Lett., 32, 407 (1995).

${ }^{8}$ J.U. Sommer and M Daoud, Phys. Rev. E, 53, 905 (1996).

${ }^{9}$ S. Lifson and B. H. Zimm, Biopolymers 1, 15 (1963).

${ }^{10}$ S. Lifson, J. Chem. Phys., 40, 3705 (1964).

${ }^{11}$ D. Poland and H. Scheraga, J. Chem. Phys., 45, 1464 (1966).

${ }^{12}$ D. Poland and H. Scheraga, Theory of Helix-Coil transition in Biopolymers, Academic Press, New York (1970).

${ }^{13}$ R. Bundschuh and T. Hwa, Phys. Rev. Lett., 83, 1479 (1999).

${ }^{14}$ W. Feller, An introduction to Probability Theory, J. Wiley, New York (1971).

${ }^{15}$ M.E. Fisher, J.Stat. Phys., 34, 667 (1984).

${ }^{16}$ C. Kittel and H. Shore, Phys. Rev., 138, A1165 (1965).

17 J. Rudnick, H. Guo and D. Jasnow, J. Stat. Phys., 41, 353 (1985).

${ }^{18}$ T. Garel, D.A. Huse, S. Leibler and H. Orland, Europhys. Lett., 8, 9 (1989).

${ }^{19}$ S. Stepanow, J.U. Sommers and I.Ya. Erukhimovich, Phys. Rev. Lett., 81, 4412 (1998).

${ }^{20}$ Z.Y. Chen, J. Chem. Phys., 111, 5603 (1999).

${ }^{21}$ V. Ganesan and H. Brenner, Europhys. Lett., 46, 43 (1999).

${ }^{22}$, A. Maritan, M.P. Riva and A. Trovato, J. Phys. A, 32, L275 (1999).

${ }^{23}$ C. Monthus, Eur. Phys. J., B, 13, 111 (2000).

${ }^{24}$ X. Châtellier and J.-F. Joanny, Eur. Phys. J., E, 1, 9 (2000).

${ }^{25}$ J. Cardy, J. Phys. A, 24, L1315 (1991). 